\title{
Understanding Implicit and Explicit Interface Tools to Perform Visual Analytics Tasks
}

\author{
Dong Hyun Jeong*, Soo-Yeon $\mathrm{Ji}^{\dagger}$, Tera Greensmith ${ }^{\ddagger}$, Byunggu $\mathrm{Yu}^{*}$ and Remco Chang ${ }^{\S}$ \\ *Department of Computer Science and Information Technology \\ University of the District of Columbia, Washington, DC 20008-1122 \\ Email: \{djeong, byu\}@udc.edu \\ ${ }^{\dagger}$ Department of Computer Science, Bowie State University, Bowie, Maryland 20715-3319 \\ Email: sji@bowiestate.edu \\ ${ }^{\ddagger}$ School of Interactive Arts + Technology, Simon Fraser University, Burnaby, Canada \\ Email: terag@sfu.ca \\ $\S$ Department of Computer Science, Tufts University, Medford, MA 02155-5801 \\ Email: remco@cs.tufts.edu
}

\begin{abstract}
Although menu systems are commonly adopted as supporting tools in visualization systems, understanding the effectiveness of supporting tools to perform visual analysis is not broadly studied. In this paper, we performed a study to see the effectiveness of implicit and explicit interface tools interactive iconic tool (Interactive-Icon) and floating text-based tool (Floating-Menu), respectively. The floating text-based tool is similar to traditional menu systems. The interactive iconic tool is designed as a direct manipulation tool for implicitly selecting objects. To evaluate the use and human performance of both tools, a highly interactive visual anlaytics system is used. With this system, a task-oriented user study is performed. In this study, participants were asked to solve six task questions, three with each tool. All participants' task performances were tracked including accuracy, speed, behaviors and post-task qualitative feedback. From the study, we identified that there is a familiarity effect on the performance of solving the task questions with implicit and explicit interface tools.
\end{abstract}

\section{INTRODUCTION}

Menu systems are commonly adopted as supporting tools to help users change system attributes or initiate new tasks. Although extensive studies on understanding the cost and benefits of pulldown menus vs. direct manipulation icons have performed in the HCI community [1], [2], [3], the distinction between the two is not nearly as clear especially in visual analytics. In this paper, we focus on understanding the effectiveness of menu systems (i.e. implicit and explicit interface tools) for solving complex analytical problems (tasks) with a highly interactive visual analytics system.

An evaluation (task-oriented user evaluation) on two menus systems, a Floating-Menu and an Interactive-Icon, is performed with a genomic visual analytics tool (called GVis [4]). The Floating-Menu supports explicit interaction that the user creates a discrete action (i.e. menu selection) and expects a timely discrete response. The Interactive-Icon is designed by following the concept of implicit interaction [5], where the user implicitly interacts with flying icons to control application settings. In the extended version of GVis, the two menus systems are embedded to evaluate which is more effective in supporting visual analysis tasks. Accuracy and speed of the analysis were quantitatively measured, and qualitative feedback on ease of use, ease of learning, preference, and effectiveness was also evaluated. During the study, each participant's interaction behavior, such as what is being viewed, and when each tool is opened, was captured in a log file. The captured log files were analyzed visually by tracking how each participant reached to the goal of finding correct answers for the given task questions.

In this paper, we will begin with exploring previous work and related research in Section II, describe GVis and the menu tools in Section III, explain the study protocol and report the results in Section IV and Section V, respectively. Our discussion and conclusion is in the last section.

\section{PREVIOUS WORK}

In visualization, interaction is regarded as an important tool that supports users analyzing data efficiently. BeaudouinLafon [6] emphasized that research focus has to be changed from designing interfaces to designing interactions. Due to the importance of supporting interactions while performing visual analysis, various visualization systems have been designed. Heer and Shneiderman [7] evaluated known interactive visualization tools by summarizing them into three high-level categories as data and view specification, view manipulation, and analysis process and provenance. Although designing interactive visualization interface tools is crucial, it is still remained as an important research challenge. In addition, identifying an effective evaluation method to determine the usefulness of the tools cannot be disregarded. Lim et al. [1] pointed out that there are limits to Norman's model, in particular the evaluation of direct manipulation interface vs. menubased interface. Although they found no time difference on performing a task, they pointed out the necessity of considering task familiarity, given instructions, and the type of interface as a part of finding the limits of Norman's model. In the area of mobile device concerns, Schröder et al. [8] tested an iconbased menu vs. a text-based menu to see the usefulness and efficiency of each system. Based on the evaluation, they found that icon-based menu system better supported learnability, but less-time was spent solving the given tasks with text-based menu. In contrary to previous research, we focus on not only understanding the differences between the tools, but also on finding factors that help the user solve a visual analytical 


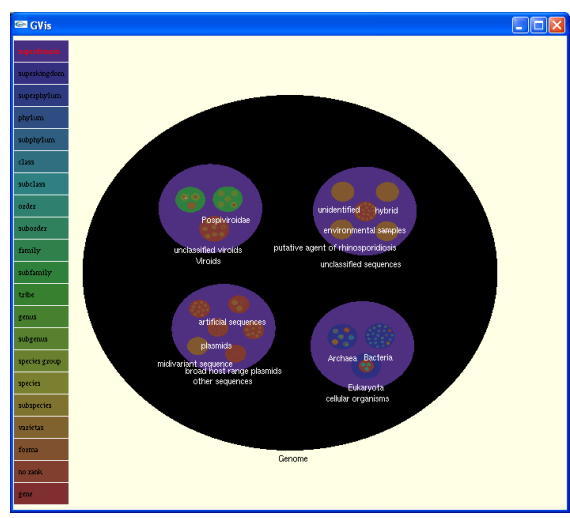

(a)

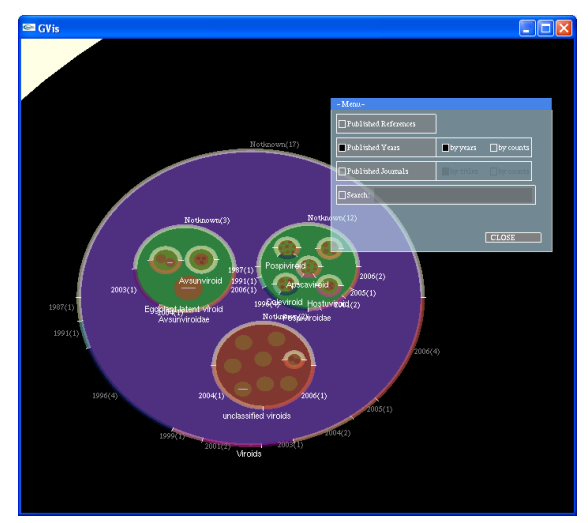

(b)

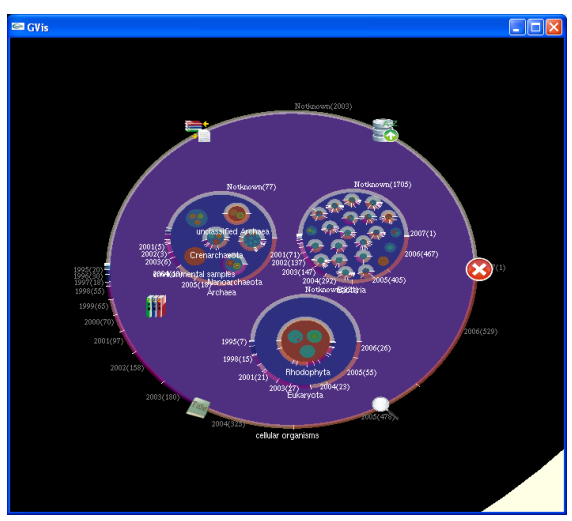

(c)

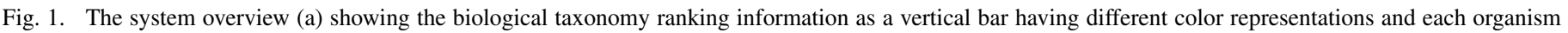

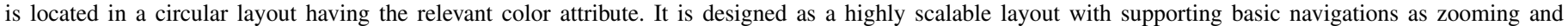
panning. (b) and (c) show two different interfaces as Floating-Menu and Interactive-Icon.

task since there is evidence that traditional menus cause considerable interruptions to an analyst's flow. Specifically, Green et al. [9] argued that visualization should avoid menus or other actions that take the user outside of the frame of the task. Since there is no known research performed to support this argument, our hypothesis of this study is to show the potential differences in effectiveness of two interface tools, one a floating text-based menu (Floating-Menu) and the other a more interactive iconic tool (Interactive- Icon).

Evaluation has a long-history in both applied psychology and computer science (in HCI). In psychology, people adopt probability and statistics as an essential tool to build knowledge of the logic of evaluation. Their studies are not only limited to understand the logic of evaluation, but also to study the domain of evaluation [10]. In computer science (especially in HCI), however, evaluation is known as a method, with which people try to find the usefulness of their designed user interfaces. There are several evaluation approaches in HCI designed and proposed by experts [2], [3]. However, a limited study has been done in the fields of information visualization and visual analytics. Since evaluation become increasingly important in visualization and visual analytics, there have been tremendous efforts in such evaluation, most notably the establishment and success of the BELIV workshops ("BEyond time and errors: novel evaLuation methods for Information Visualization" [11], [12]). Active research topics in this area might include the evaluation of techniques, the measurement of insights, and metrics and methodologies for user studies. Together, these efforts are gradually validating the usefulness and power behind the science of information visualization and visual analytics. In BELIV' 06 workshop, specifically, many distinctive approaches are proposed. For instance, Rester and Pohl applied different methods to evaluate a visualization called Gravi++ that includes the use of insight reports [13]. Shneiderman and Plaisant [14] emphasize the importance of Multi-dimensional In-depth Long-term Case studies (MILCs) as a new evaluation method for information visualization applications. Their method is a new paradigm to analyze information visualizations in cooperating with domain experts. However, longitudinal evaluation is not commonly accepted in visualization because it requires a period of several weeks to several months to validate. Later, in BELIV'08 [12] and BELIV'10 [15] workshop, visualization experts discussed current limitations of evaluating information visualizations. Although most researchers in information visualization and visual analytics are agreed that evaluation is important and necessary, how to evaluate visualizations is not broadly studied. Since limited study has been done in evaluating visualizations, we perform our evaluation study based on a task-oriented user evaluation method (e.g. user-centered evaluation [12]).

\section{SYSTEM}

\section{A. GVis (Genomic Visualization)}

Within a genomic visualization (called GVis [4]), we tested implicit and explicit interface tools - floating text-based tool (Floating-Menu) and interactive iconic tool (InteractiveIcon). GVis [4] is an expert visualization system that helps bioinformaticians to support the visual analysis of large-scale phylogeny hierarchies populated with the genomic data of various organisms. It uses a publicly available biological database (GenBank) hosted by the National Center for Biotechnology Information (NCBI, http://www.ncbi.nlm.nih.gov) to picture the phylogeny hierarchies of organisms and allows the user to quickly browse the hierarchy from the highest-level, base categorization down to the level of individual genome for the desired organism of interest. The most current version of GVis was used in this study, which utilizes a more robust ontological-like structure than the versions before it.

Figure 1(a) shows a system overview; phylogenic hierarchies are represented in spheres, and each organism is directly mapped within that sphere. On top of GVis, two interface tools were designed: Floating-Menu and Interactive-Icon. Based the chosen option, relevant information is represented as shown in Figure 1(b) and (c). However, representing 18,000 citations, in addition to the organismic information in a limited display space is not feasible. Therefore, an aggregation technique was utilized to make the visualization both complete and scalable. If an organism is located in the lower level of the phylogeny, the represented information is too small to be perceived; aggregation is then automatically applied to show such information within the organization of the higher categorizational level. Figure 1(b) and (c) shows related citations grouped by year and organism. 


\section{B. Floating-Menu Tool}

Because of data complexity, most visual analytics applications commonly adopt traditional text-based menus (e.g. pull-down, pop-up) to support changing displays views and system functionality. Figure 2 shows four commonly used menu systems in visual analytics applications where a gray background indicates visual space and the objects in the visual space represent visual glyphs. The pull-down menu system (Figure 2(a)) is a common technique which statically positions the menu on the window's title bar. Clicking the menu causes menu items to appear to drop down from it. Although it is a simple and broadly used menu system, the menu can become illegibly small in a scalable, complex visualization. The menu-button system uses several buttons positioned internally (Figure 2(b)) or externally (Figure 2(c)) in a main window. Both internally and externally positioned menu-buttons are useful, intuitively showing the available options. However, the internally positioned menu-buttons always occupy some visual space. Similar to the pull-down menu system, both menu-button systems cause a limitation of interacting with if the scale of the visual space is enlarged. The pop-up menu system (Figure 2(d)) addresses the limitation existed in other menu systems by directly mapping menu-options with a visual object. GVis' Floating-Menu is designed to act similarly the pop-up menu system, but without linking the menu to a static object, the menu "floats" over the visual space, and can be easily grabbed and moved out of the way. Additionally, its background is translucent, in an attempt to prevent it from obscuring the field of view.

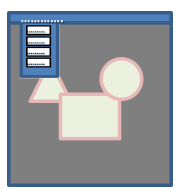

(a)

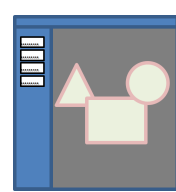

(b)

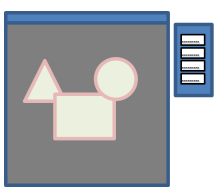

(c)

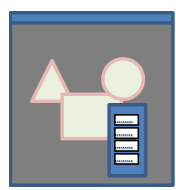

(d)
Fig. 2. A representation of commonly used menu systems in visual analytics applications.

The Floating-Menu allows access to four core functionalities, which can be utilized one at a time (see Figure 1(b)). The first option ('Published References')visualizes the number of related published and unpublished journal articles. The second ('Published Year') provides the years in which journals published articles about an organism. The third ('Published Journals') visualizes the names of the publishing journals. And the fourth ('Search:')is a Boolean text-based search. The second and third options can be combined through use of sorting options that are positioned next to each one. Based on the sorting option, information is sorted by published year/ number of publications in each year, and the names of the journals in alphabetical order, and the number of publications relevant to each journal.

\section{Interactive-Icon Tool}

The Interactive-Icon seeks to minimize the use of text and the need for a separate menu. It uses six icons, each placed around the circumference of the organism's sphere (see Figure 1(c)). Unlike the Floating-Menu's radio buttons, the icons are dragged and dropped to the area of interest. For
TABLE I. THE FIVE ICONS USED IN INTERACTIVE-ICON

\begin{tabular}{|c|c|c|}
\hline Icon & \multicolumn{2}{|c|}{ Meaning } \\
\hline$\sqrt{2}$ & \multicolumn{2}{|c|}{ Published/ Unpublished papers } \\
\hline IIII & \multicolumn{2}{|l|}{ Published years } \\
\hline ingeg & \multicolumn{2}{|l|}{ Journal titles } \\
\hline$\nabla$ & \multicolumn{2}{|l|}{ Searching } \\
\hline 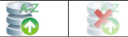 & Sorting (Possible) & Sorting (Not possible) \\
\hline$x$ & \multicolumn{2}{|l|}{ Cancelation } \\
\hline
\end{tabular}

example, if the user wants to see the relevant information related to organism $\mathrm{A}$, then an icon needs to be dropped in the region between the organism A and its subgroup organism(s). However, each icon does not represent data itself nor its symbolic meaning the way traditional icons do. Instead, it represents an operation to be performed. The icons can be dragged to active the same four core functionalities as the Floating-Menu. If the represented information can be sorted, the icon is shown as active; otherwise, it bears an " $\mathrm{x}$ " indicating its unavailability (see Table I). The cancellation icon, when dragged and dropped, returns to the visualization's previous state so another function can be used. Additionally, the visualization can be returned to its original view by pulling the icon back into the sphere's circumference.

In a scalable visualization, the user's focus is always changeable. Therefore, the Interactive-Icon adopts a focusdependent representation method. The icons are automatically mapped on circumference of interest. Also Interactive-Icon has an auto-hide feature that automatically hides the icons when navigating the larger genomic space in order to minimize the visualized clutter.

\section{Representing Information}

As mentioned above, each sphere represents an organism and biological taxonomy (such as domain, kingdom, etc. [16]). Each organism's name color attribute is determined based on its biological taxonomy. Figure 1(a) shows the initial system layout with each organism's name positioned below and a color-coordinated biological taxonomy located on the left side of the view. Whenever an option or icon is selected in either the Floating-Menu or the Interactive-Icon, the relevant information is represented around the organism circle circumference.

Figure 3 demonstrates how the each core functionality visualizes new information. As mentioned previously, all information is automatically aggregated before it is represented. The number of published and unpublished journal articles related to each organism is shown in Figure 3(a). Published papers are highlighted in yellow, unpublished in green. Figure 3(b) and (c) show more complex data, such as publication year and the title of the publishing journal with textual information. Tone mapping is used to delineate information. For example, Figure 3(d) highlights the number and location of search results by using a different line thickness in a high-contrast blue.

\section{COMParative StUdy}

We conducted a within-subject study to explore which interface was more effective. 31 participants (twelve males and nineteen females) performed a total six performance tasks, 3 with each interface. 


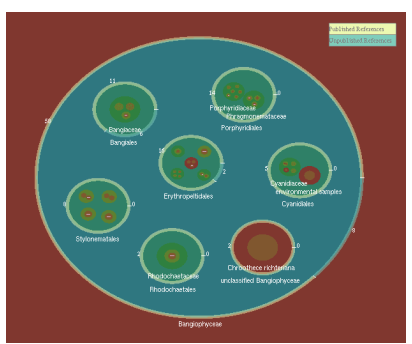

(a)

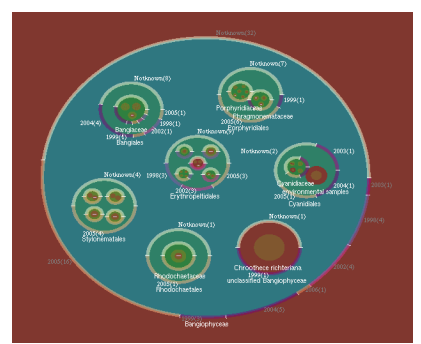

(b)

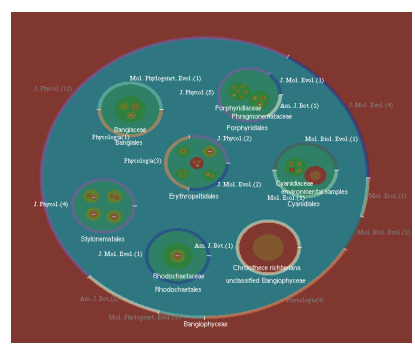

(c)

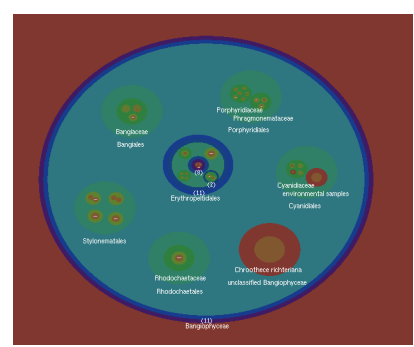

(d)

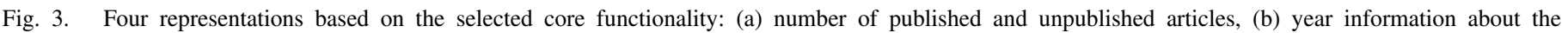
publications, (c) titles of the publishing journals, and (d) result of a search.

\section{A. Procedure}

Prior to beginning the each of the two task sets, participants were given a tutorial about the tool they were about to use, including how to utilize basic functionalities. Then, the participant was requested to answer four training questions with the tool; these questions were designed to introduce participants to essential functionalities of the tool. After the training, the evaluation was conducted. The participants were given a plenty of time (no time limit) to perform each task and were requested to answer questions immediately after each task. The evaluation was conducted using an online website, where time spent and answers were saved into a database.

Two task sets were designed to measure the effectiveness when using the two interface tools. The order in which the participants used each tool was counterbalanced. The task sets were carefully created to maintain the same levels of difficulties across the sets: the first question in each set is easy to solve, the second question is a bit difficult, and the last question was designed to be the most difficult.

Two sets of task questions are used:

[SET-1]

- Count how many papers were A) published and B) not published about the group Bacteria.

- $\quad$ Find the organism whose one research paper was published in 2007.

- This question has three parts.

1) First, count how many publications were published about group Rhizobiales.

2) Secondly, count the number of publication(s) for Rhizobiales in 1995.

3) Thirdly, name the journal that published 7 papers in total about Rhizobiales.

\section{[SET-2]}

- How many papers in total were published about the Apscaviroid group?

- Name the organism whose research paper(s) were published in 1987.

- There were multiple papers published in 2006 about the Micrococcineae (This questions has 2 parts).

1) First, how many papers are published in 2006?

2) And what is name of the journal that published 11 of those papers?
Several outcome variables were tracked throughout the study, which include task completion time, accuracy, and interaction behaviors. Incorrect answers to the task questions were counted to measure the accuracy. Each participant's timestamped user interactions were captured by built-in functions of the system and saved automatically into a log-file to attempt to determine each participant's flow of cognition. A post-task questionnaire was administered to track qualitative feedback about each task question. These questions included "how difficult was the task question?" and "how helpful was the interface to solving the task question?" Additionally, at the end of the evaluation of each tool, a post-interface questionnaire was administered. This questionnaire asked the participant to evaluate the ease of learning and ease of use of each interface. And, after a participant completed the evaluation of both tools, a post-study questionnaire was administered, which requested feedback on the comparative ease of use of both tools, including open-response questions about participant likes and dislikes. The last questions asked the participant to grade each tool, on a scale from 'A' to ' $F$ '.

\section{Study Results}

\section{A. Quantitative Analysis}

Accuracy: Approximately $54.84 \%(17.0 \pm 7.9)$ of the participants answered correctly using Floating-Menu. On the other hand, when using Interactive-Icon, about $61 \%(14.3 \pm 5.0)$ of them were able to answer correctly. Furthermore, there were two instances in which participants could not complete the task when using Floating-Menu and three instances when using Interactive-Icon. By looking at the answers and the captured log-file, we identified that some participants (9 participants in Floating-Menu and 12 participants in Interactive-Icon) got close to the goal, but they did not answer correctly. When analyzing the result, we considered the accuracy with and without a half-point given for this. Based on the statistical analysis, we found that the accuracy difference is not statistically significant across the two interfaces with $(p=0.23)$ and without $(p=0.24)$ a half point given. Interestingly, we found that the accuracy difference is statistically significant across the gender with $(p=.0039, F(1,185)=8.55, R 2=.044)$ and without $(p=.0013, F(1,185)=10.69, R 2=.0054)$ a half point given. About $69.4 \%$ of male participants correctly solved the given tasks; whereas only about $50.4 \%$ of female participants provided correct answers. Rohr [17] conducted a study to see the gender difference when performing a computer-pointing task. From the study, she found that women perform better 
when accuracy is analyzed. Beckwith et al. [18] showed that there is a self-efficacy difference between male and female. However, they described that there are not only self-efficacy ${ }^{1}$, but also motivation, problem-solving styles, learning styles, and information-processing styles are all implicated in gender differences.

Speed: During the training session, participants spent about 6 minutes on average to become familiar with the application as well as the interfaces. Table II shows the overall average time spent solving each task. Participants spent more time in solving the last task when using each tool. This seems to be because the task involves more than two subquestions. On average, participants spent about 174 seconds using Floating-Menu, and 204 seconds using Interactive-Icon. Interestingly, the average time spent when performing task 1 with Interactive-Icon shows that the participants spent less time than when using Floating-Menu (see Table II). In this study, we assume that the user might spend less time when solving the given tasks with an interactive interface tool. However, we found no statistically significant difference $(p<0.25)$ on timespent. In addition, there was no gender difference. Although men perform better to the tasks, where completion time is mainly concerned [17], our result does not support this claim.

TABLE II. AVERAGE TIME SPENT (SECONDS) IN SOLVING EACH TASK.

\begin{tabular}{|l|l|l|l|l|}
\hline \multicolumn{2}{|c|}{} & Male & Female & Total (Average) \\
\hline \multirow{3}{*}{ Floating-Menu } & Task1 & 175.16 & 94.52 & 125.74 \\
\cline { 2 - 5 } & Task2 & 150.91 & 102.05 & 120.96 \\
\cline { 2 - 5 } & Task3 & 209.75 & 319.84 & 277.22 \\
\hline \multirow{3}{*}{ Interactive-Icon } & Task1 & 103.50 & 83.68 & 91.35 \\
\cline { 2 - 5 } & Task2 & 186.58 & 164.78 & 173.22 \\
\cline { 2 - 5 } & Task3 & 338.83 & 353.78 & 348.00 \\
\hline \multicolumn{2}{|l|}{ Total (Average) } & 194.12 & 186.44 & 189.41 \\
\hline
\end{tabular}

By measuring least square means about the accuracy (Figure 4(a)) and the time spent (Figure 4(a)), participants spent more time when solving more difficult task questions (difficulty of each task: task $1<$ task $2<$ task 3). Based on Pearson's Correlation Coefficient measure, there is a trend $(r=.47, p<.0001)$ between the time spent and the difficulty of the task. Although it is not statistically significant $(r=$ $.12, p=0.09)$, there is a negative correlation between between the accuracy and the difficulty of the task. The participants spent less time with Interactive-Icon for solving the easy task (task 1), the accuracy is lower than with Floating-Menu. However, when solving the difficult task (task 3), the accuracy was slightly, but not significantly higher with Interactive-Icon even if they spent significantly more time.

\section{B. Qualitative Analysis}

Easiness \& helpfulness (post-task questionnaire): Participants are requested to report the easiness and helpfulness of the interface in solving the task. About 60\% (18.6 \pm 0.5 for each task) of the participants reported all 3 tasks to be "easy" or "very easy" when using Floating-Menu. On the other hand, only about $43 \%(13.3 \pm 4.0$ for each task) of the participants identified the tasks as being "easy" or "very easy" when using Interactive-Icon. Interestingly, about 60 percent (in average) of the participants mentioned that all the tasks are easy to solve

\footnotetext{
${ }^{1}$ Self-efficacy: The belief that one is capable of executing certain behaviors or reaching certain goals.
}

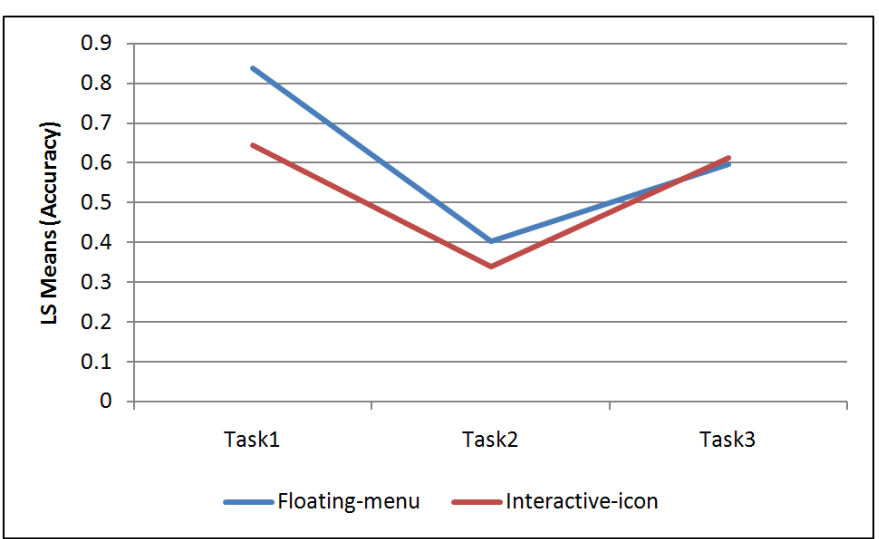

(a)

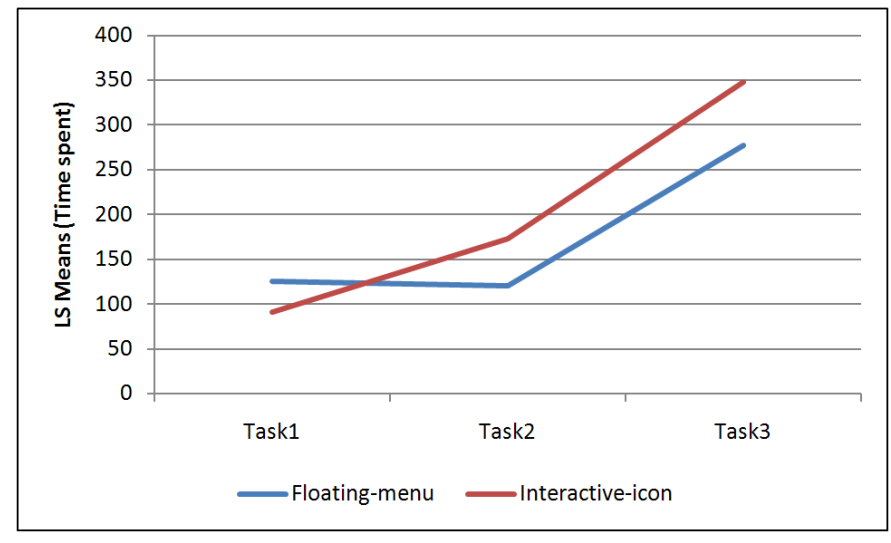

(b)

Fig. 4. Least Square Means of (a) Accuracy and (b) Time spent (seconds) when solving each task. In (a), the accuracy value close to 1 indicates the participant found a correct answer.

when using Floating-Menu, but the accuracy in solving each task is low for task 2 and task 3 (below average); whereas about $43 \%$ (in average) of the participants mentioned that the tasks are easy to solve when using Interactive-Icon, only task 2 has low accuracy (below average). This might be because most participants might have experienced with the menu-based system, and have little previous experience with the interactive interface. Furthermore, only about $58 \%$ (for Floating-Menu) and $29 \%$ (for Interactive-Icon) of the participants indicated that task 3 is easy; whereas the accuracy is up to $70 \%(68.52 \%$ and $70.37 \%$ respectively). Since task 3 has multiple subquestions lead to creating several continuous interactions when using Interactive-Icon, the higher cost of interaction might make participants feel the task is not easy. Interestingly, a participant (P11) indicated that all tasks are difficult to solve using Floating-Menu; whereas two participants (P26 and P29) rated that all tasks are difficult to solve using Interactive-Icon.

About $74 \%(23 \pm 3.6$ for each task) of the participants identified Floating-Menu to be "helpful" or "very helpful" in solving the tasks, and about $65 \%$ (20 \pm 3.6 for each task) of the participants found Interactive-Icon to be "helpful" or "very helpful." Furthermore, two participants (P10 and P11) indicated both interfaces to be not helpful in solving tasks.

Overall, we find that the more easy a task was rated (very easy $=5$, easy $=4$, etc), the less time the participants spent on 
solving it $(r(185)=-.26, p<.0002)$. Also, solving a task with a "helpful" interface (very helpful $=5$, helpful $=4$, etc.) decreases the time spent on the task $(r(185)=-.26, p<$ .0002). With a easy and helpful interface, the participants solved the tasks more accurately $(r(185)=.401, p<.0001$ for easiness and $r(185)=.43, p<.0001$ for helpfulness).

Learnability (post-application questionnaire): From the post-application questionnaire, about $67 \%$ and $51 \%$ of the participants rated that Floating-Menu and Interactive-Icon were easy to use ("very easy" or "easy"), respectively. $70 \%$ of the participants mentioned Floating-Menu was very easy or easy to learn; whereas $58 \%$ of the participants rated that Interactive-Icon was very easy or easy. Furthermore, one participant indicated that Interactive-Icon is very difficult to learn how to use. Since all participants were not familiar with interactive visual interface, they felt simple selection mechanism in Floating-Menu was much easier to learn than Interactive-Icon. These might be because user's familiarity is quite related to determining ease of learning and ease of use on each interface. All the participants' daily usage of computers (96\% of the participants claimed that they uses a computer almost everyday) indicates that they are already familiar with windows-based menu systems.

Based on the analysis using Pearson's Correlation Coefficient measure, ease of use and ease of learning are examined. The participants' accuracy revealed a positive correlation (Ease of use: $r(185)=.21, p=.0035$ and ease of learning: $r(185)=.28, p=.0001)$. However, the overall time spent in solving each task showed no statistically significant correlation (Ease of use: $p=.174$ and ease of learning: $p=.429$ ).

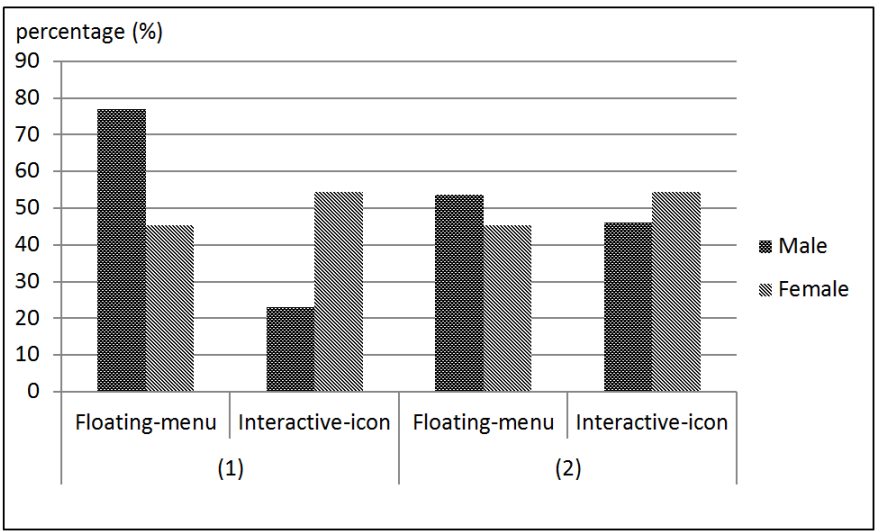

Fig. 5. Gender differences on (1) Which interface did you like better? and (2) Which interface did you feel more comfortable?

Preference (post-study questionnaire): After the evaluation, each participant ranked the two interfaces, provided their preferences, and described their pros and cons. Based on the participants' preferences, we found that there is no significant difference on the participants' preference over Floating-Menu and Interactive-Icon. However, we found that there is some gender difference in their preferences. Majority of the female participants felt Interactive-Icon is better and more comfortable to use than Floating-Menu (Figure 5).

Based on the description of the pros and cons, we found that several participants pointed out the strength of InteractiveIcon as being "interactive". Two participants mentioned that "a good feature of Interactive-Icon is adopting a hands-on approach that helps bringing with me wherever I am navigating through the zoomable genomic space." Furthermore, six participants specifically pointed out that moving icons help them to directly focus on the organism without causing any attentional interference [19] by the icons. However, one participant (P3) commented that he continuously forgot the meaning of each icon and suggested providing the name of each icon might be useful to recall the meaning of icons.

For Floating-Menu, three participants indicated that it is direct, straight forward to use, and easy to be familiar with. On the other hand, two participants commented that the listed options are complicated. One participant (P19) pointed out she continuously forgot which option was to be used to solve the given task question. One participant (P8) complained that Floating-Menu has to be moved out of the way from his focus of interest because it occludes the represented results that he is searching for. Overall, the participants did not mention a lot of pros in Floating-Menu. This might be because the design mechanism used in Floating-Menu is the same as the menu system that they continuously see in their daily computer use.

\section{Factor Analysis}

Based on Lam's seven costs [20], we found that both interface tools do not commonly share the three costs such as physical motion cost to execute sequences, visual-cluttering cost to perceive state, and view-change cost to interpret perception. The physical-motion cost is the cost to form physical sequences. As an implicit interface tool, the Interactive-Icon requires mouse dragging cost. As an explicit interface tool, an accumulated motion cost (mouse clicks to select an option(s)) might occur in the Floating-Menu. Although both tools cause visual-cluttering problem when the represented information is blocked by a menu and icons, each tool has a different value of the visual-cluttering cost that can simply be measured by the area size of each tool. If the visual-cluttering cost is high, users might have difficulty perceiving the represented information. However, since the icons in the Interaction-icon tool are designed as small, the user's focus of interest cannot easily be interfered by visual occlusion and distraction. Table III shows the interaction costs that each interface tool might occupy.

TABLE III. A SUMMARY OF INTERACTION COSTS EXISTS IN EACH INTERFACE TOOL.

\begin{tabular}{|l|l|l|}
\hline & Floating-Menu & Interactive-Icon \\
\hline Physical motion cost & Low & High \\
\hline Visual-cluttering cost & High & Low \\
\hline View-change cost & High & Low \\
\hline
\end{tabular}

In GVis, a zooming interaction is performed by simply clicking on mouse buttons. The button clicks might produce the physical motion cost (the accumulated motion cost). However, the accumulated motion cost caused by the zooming is greater than the cost created when clicking options. Overall, the Interactive-Icon has low visual-cluttering and view-change costs; whereas the Floating-Menu has only low physical motion cost (see Table III).

Lim et al. [1] pointed out that the task performance is quite related with the user's familiarity as well as automaticity. Zuilova-Seinstra et al. [21] mentioned that "The sophistication 
of visualization techniques used or created depend largely on the level of expertise and familiarity of the technology and its uptake." Since about $96 \%$ of the participants are new to the visualization application as well as the new interface (Interactive-Icon), we believe that the familiarity might be an issue that causing no significant difference between the tools. Since the Floating-Menu adopts representing words in the menu system, we also look at the effectiveness of the reading automaticity [22]. Although there is no existing literature of understanding the effectiveness of the text-based menu versus iconic tool in considering the reading automaticity, it has long been believed that reading words is an unconscious action [22]. Based on this belief, we believe that the reading automaticity can be a factor that reduces the reaction time when solving the tasks.

TABLE IV. A RESULT FROM ANALYZING THE INTERACTION LOGS.

\begin{tabular}{|l|l|l|}
\hline & Floating-Menu & Interactive-Icon \\
\hline Attentional interference (ea) & 3.1 & 17.8 \\
\hline Activation duration (second) & 443.35 & 261.37 \\
\hline
\end{tabular}

Finally, we analyzed the interaction $\log$ s to see the cognitive flow differences. Figure 6 shows two participants interaction logs. Each dot indicates what organism the participant was looking at. The background colors (blue and red) represent which tool is set visible: blue (the Interactive-Icon) and red (the Floating-Menu). The $\mathrm{x}$-axis represents time and the $\mathrm{y}$ axis indicates depths of the taxonomically displayed genomic information. We found that the participants create two different patterns when using the Floating-Menu. Figure 6(a) shows that the participant solved the task questions with the FloatingMenu visible (see the red-color region D in Figure 6(a)); whereas Figure 6(b) shows that the participant was continuously making the Floating-Menu visible and invisible (see the jagged red-color region B in Figure 6(b)). We simply assume that users will use each tool and make it visible when they want to change the options. However, the interaction logs explain that some participants did opposite.

With the interaction logs, we analyzed users' attentional interference and activation duration (see Table IV). The average attentional interference indicates that how many times the participants are being intentionally or unintentionally interrupted by each tool. The average activation duration represents how long each tool has been displayed on screen (and watched by the participants). Based on statistical analysis, we found that the attentional interference $(p=.0005, F(1,39)=14.48$, $\left.R^{2}=.275\right)$ and activation duration time $(p=.0091$, $\left.F(1,39)=7.55, R^{2}=.166\right)$ are statistically significant across the tool. Also there is a negative correlation coefficient between the accuracy and the interruptions $(r(40)=-.334$, $p=.035)$. However, we did not find a significant correlation in the accuracy across the activation duration time $(p=.31)$. As we mentioned above, each tool occupies some visual space; therefore the user has to change her focus on between the tool and the genomic space when solving the tasks. If the participant continues performing the task without making the tool disappeared, it might deliberately interrupt the participant.

\section{DISCUSSION AND CONCLUSION}

Although Lim et al. [1] conducted the evaluation on trivial tasks in a simple testing environment about almost two decades

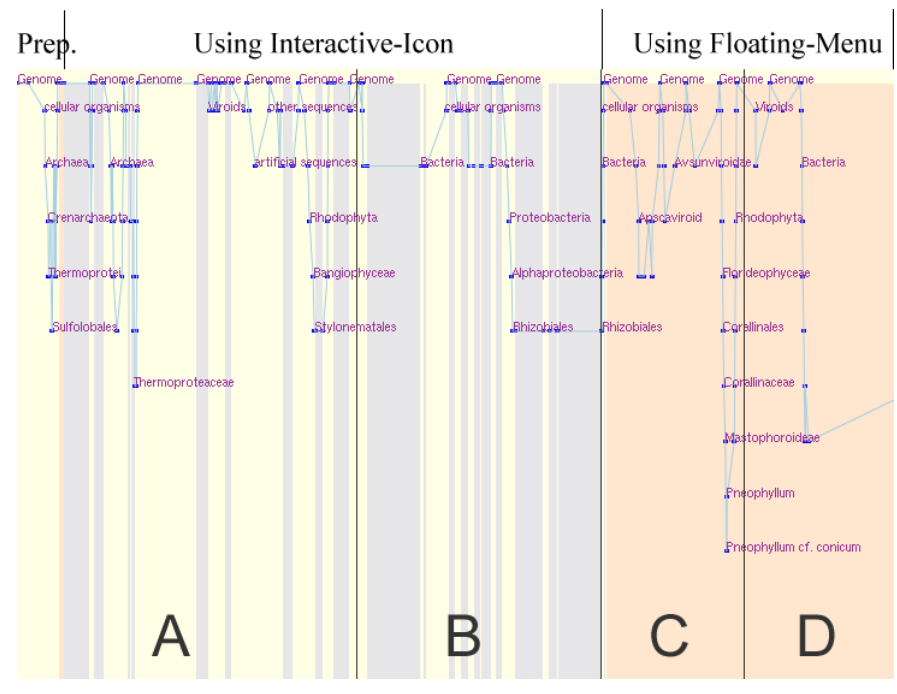

(a)

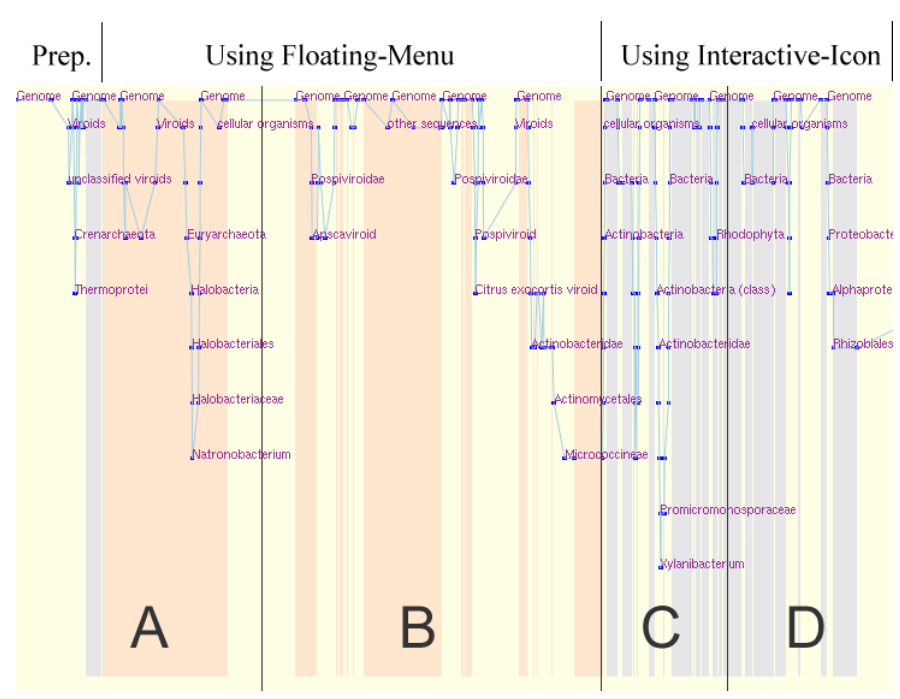

(b)

Fig. 6. Two participants' interaction logs. Colored backgrounds indicate the activation of Interactive-Icon and Floating-Menu. The figures indicate that the participant used the Interactive-Icon first in (a) and the other participant used the Floating-Menu first in (b). Section A and C indicate the interactions created during the training session. And section B and D represent the participants' interactions when solving the tasks.

ago, their findings may be generalizable in some ways to complex analysis processes using visual analytical tools. The claim by Green et al. [9] refers to menus causing interruptions to an analyst's cognitive flow in terms of gathering usable knowledge and perhaps discovering new insights for the task at hand. Of course, it is important to examine if the variables we are measuring do indeed reflect the flow of cognition and the building of usable knowledge. We believe that the claim is made under the assumption that the visualization is being used by either experts or knowledgeable people who are familiar with the task at hand. Indeed, this assumption is basic to the understanding of what cognitive flow is. Both Lim et al.'s experiment and ours used students as participants, which may preclude the cognitive flow characteristic of this type of reasoning without some training and experience. The 
evaluation must be performed with visualizations and tasks that are familiar to knowledgeable users. In fact, Lim et al. noted that in a study in which participants are familiar with the task, direct manipulation icons do lead to faster task performance. Given how closely our evaluation has echoed Lim et al.'s findings, we hypothesize that, should we repeat the process with actual experts, we might likely find that Interactive-Icon outperform Floating-Menu.

In [1], Lim et al. found a significant difference in the user's performance between executing familiar and less-familiar tasks. Based on the study of the concept of familiarity, de Walle et al. [23] found that most users have familiarity with menu-based interfaces. It is important to consider the learning curve involved in using a novel interface, especially when the participants needs to learn not just the interface but a visual analytical tool and a task in the bioinformatics domain. The potential of interface familiarity as a confounding factor further highlights the need to use knowledgeable users and more complex tasks in order to reduce learning factors as much as possible or conversely to do experiments with appropriate training built in. Automaticity is defined as the ability to do things without occupying the mind with the low level details [22]. Since automaticity affects nearly every aspects of our everyday life, psychologists have studied its effectiveness in great detail. Since, in our experiments, all tasks require reading annotated text information, reading automaticity should be considered. Lastly, attentional interference might be an important consideration. Task performance is subject to both intentional and unintentional interruptions. If participants are interrupted often it will affect the flow of cognition and degrade the overall performance of solving a task.

The experiments, as well as the considerations and analysis they engendered in this paper have set the stage for significant new work. Based on this analysis, we and others can now undertake deeper and more careful experiments on the relationships between the use of interactive tools during engaged cognitive flow and reasoning processes. This is a central research issue for visual analytics. For future works, we plan to extend our study to identify factors that interrupt the flow of cognition while performing a visual analysis.

\section{ACKNOWLEDGMENT}

This work was partially supported by US Army Research Office (ARO) grant W911NF13110143.

\section{REFERENCES}

[1] K. H. Lim, I. Benbasat, and P. A. Todd, "An experimental investigation of the interactive effects of interface style, instructions, and task familiarity on user performance," ACM Trans. Comput.-Hum. Interact., vol. 3, no. 1, pp. 1-37, 1996.

[2] B. E. John, "Evaluating usability evaluation techniques," ACM Comput. Surv., vol. 28, p. 139, 1996.

[3] B. Shneiderman and C. Plaisant, Designing the User Interface: Strategies for Effective Human-Computer Interaction, 4th ed. Addison Wesley, April 2004.

[4] J. Hong, D. H. Jeong, C. D. Shaw, W. Ribarsky, M. Borodovsky, and C. G. Song, "Gvis: A scalable visualization framework for genomic data." in EuroVis, K. Brodlie, D. J. Duke, and K. I. Joy, Eds. Eurographics Association, 2005, pp. 191-198.

[5] A. Schmidt.
[6] M. Beaudouin-Lafon, "Designing interaction, not interfaces," in AVI '04: Proceedings of the working conference on Advanced visual interfaces. New York, NY, USA: ACM, 2004, pp. 15-22.

[7] J. Heer and B. Shneiderman, "Interactive dynamics for visual analysis," Commun. ACM, vol. 55, no. 4, pp. 45-54, Apr. 2012.

[8] S. Schröder and M. Ziefle, "Making a completely icon-based menu in mobile devices to become true: a user-centered design approach for its development,' in MobileHCI '08: Proceedings of the 10th international conference on Human computer interaction with mobile devices and services. New York, NY, USA: ACM, 2008, pp. 137-146.

[9] T. Green, W. Ribarsky, and B. Fisher, "Visual analytics for complex concepts using a human cognition model," in Visual Analytics Science and Technology, 2008. VAST '08. IEEE Symposium on, Oct. 2008, pp. 91-98.

[10] M. Scriven, "The nature of evaluation part i: relation to psychology," Practical Assessment, Research \& Evaluation, vol. 6, no. 11, pp. 11491156, 1999.

[11] BELIV '06: Proceedings of the 2006 AVI workshop on BEyond time and errors: novel evaLuation methods for Information Visualization, 2006, conference Chairs-Enrico Bertini, Catherine Plaisant, and Giuseppe Santucci.

[12] BELIV '08: Proceedings of the 2008 AVI workshop on BEyond time and errors: novel evaLuation methods for Information Visualization, 2008, conference Chairs-Enrico Bertini, Adam Perer, Catherine Plaisant, and Giuseppe Santucci.

[13] M. Rester and M. Pohl, "Methods for the evaluation of an interactive infovis tool supporting exploratory reasoning processes," in BELIV '06: Proceedings of the 2006 AVI workshop on BEyond time and errors: novel evaLuation methods for Information Visualization, 2006, pp. 1-6.

[14] B. Shneiderman and C. Plaisant, "Strategies for evaluating information visualization tools: multi-dimensional in-depth long-term case studies," in BELIV '06: Proceedings of the 2006 AVI workshop on BEyond time and errors. New York, NY, USA: ACM, 2006, pp. 1-7.

[15] BELIV '10: Proceedings of the 2010 AVI workshop on BEyond time and errors: novel evaLuation methods for Information Visualization, 2010, conference Chairs-Enrico Bertini, Adam Perer, and Heidi Lam.

[16] D. L. Wheeler, C. Chappey, A. Lash, D. Leipe, T. Madden, G. Schuler, T. Tatusova, and B. Rapp, "Database resources of the national center for biotechnology information," Computer, vol. 28, no. 1, pp. 173-180, 2006

[17] L. E. Rohr, "Gender-specific movement strategies using a computerpointing task." Journal of Motor Behavior, vol. 38, no. 6, pp. 431 437, 2006.

[18] L. Beckwith, M. Burnett, V. Grigoreanu, and S. Wiedenbeck, "Gender hci: What about the software?" Computer, vol. 39, no. 11, pp. 97-101, 2006.

[19] B. L. Harrison, H. Ishii, K. J. Vicente, and W. A. S. Buxton, "Transparent layered user interfaces: An evaluation of a display design to enhance focused and divided attention," in Proceedings of the SIGCHI Conference on Human Factors in Computing Systems, ser. CHI '95, 1995, pp. 317-324.

[20] H. Lam, "A framework of interaction costs in information visualization," Visualization and Computer Graphics, IEEE Transactions on, vol. 14, no. 6, pp. 1149-1156, Nov.-Dec. 2008.

[21] E. Zudilova-Seinstra, T. Adriaansen, and R. v. Liere, Trends in Interactive Visualization: State-of-the-Art Survey. Springer Publishing Company, Incorporated, 2008.

[22] A. Baddeley and L. Weiskrantz, Eds., Attention: Selection, Awareness, and Control: A Tribute to Donald Broadbent. Oxford University Press, 1995.

[23] G. van de Walle, P. Turner, and E. Davenport, "A study of familiarity," in Human-Computer Interaction - INTERACT'03, 2003, pp. 463-470 